\title{
From the journals
}

\section{Association of systolic hypertension with complications of type 2 diabetes}

It is known that diabetics who have hypertension are more likely to develop complications than those who have not, and that these complications can be reduced by treating the hypertension properly. The UKPDS 36 study results confirm this. Each $10 \mathrm{mmHg}$ reduction in systolic BP is associated with reduction in risk of $12 \%$ for all complications related to diabetes, $15 \%$ for deaths related to diabetes, $11 \%$ for myocardial infarction and $13 \%$ for microvascular complications. Lower the blood pressure, lower the risk of complications. British Medical Journal 2000; 321: 412-9. There is a large patient population in Sri Lanka with both these diseases. The message for both clinicians and patients is to strive for good control of blood pressure.

\section{MMR vaccine and allergy}

The MMR vaccine is now increasingly used in Sri Lanka, and some information about the allergic reactions seen with this vaccine is available. In the UK, between 1963 and 1997, 55 cases of anaphylaxis and anaphylactoid reactions and 8 cases of angioedema have been reported. In Japan 366 cases of allergy to MMR vaccine (34 of which were anaphylactoid reactions) have been reported from 1994 to 1997 . Egg-related antigens, gelatin added as a stabiliser and neomycin present in the vaccine are thought to be responsible. Recent studies have shown that children with egg allergy do not appear to be at increased risk of anaphylaxis after MMR vaccination. If there are any concerns specialist advice should be sought to administer the vaccine under controlled conditions in hospital. The general recommendation is that "egg allergy is not what really counts". Rather, all vaccinations - not just MMR - must be carried out in settings with equipment and trained people to deal with anaphylaxis. Archives of Disease in Childhood 2000; 82: 93-5.

\section{The risks of having children in later life}

Advancing age strongly increases the chances of three untoward outcomes in pregnancy - stillbirth, miscarriage and ectopic pregnancy. Stillbirths associated with advanced maternal age may be attributed to greater incidence of chromosomal abnormalities, pre-eclampsia and diabetes. Though the relative risk for older women is a concern, the absolute rate of stillbirths for 1000 live births has steadily declined over the past 30 years. Older women have consistently increased risk of Down syndrome, which is detectable now by prenatal screening. In addition, older women are more likely to have difficulty in conceiving and in sustaining an embryo. Women in the late 40 s seldom conceive and complete a pregnancy without the assistance of modern techniques. Some rare disorders such as achondroplasia are more common among births to older fathers. Despite the biological disadvantages experienced by older parents some studies have shown that children born to older parents do better at school than those of very young parents. It may be that older parents have greater knowledge and experience, and are often in a better economic situation, so that child rearing is more affordable. Biological disadvantage is to a degree balanced by social advantages. British Medical Journal 2000; 320: 1681-2.

\section{Preventing dog bites in children}

Dog bite is a major cause of injury, particularly in children. Researchers from Australia have tried out the effect of an educational program (called "Prevent-a-bite") directed at primary schoolchildren aiming to instil precautionary behaviour to reduce the incidence of attacks. A randomised controlled trial of its efficacy was done in 8 primary schools, including 346 children aged 7 to 8 years. The intervention group received a 30-minute lesson conducted by a dog handler highlighting the dos and don'ts of behaviour near dogs. The two groups were allowed to play unsupervised in the school grounds and a docile labrador was tethered 5 metres away from its owner disguised as a tradesman. The behaviour of children was video-taped by a hidden camera for 10 minutes. Children who received the intervention showed appreciably greater precautionary behaviour than those in control schools. In contrast, most of the children in control schools patted the dog without hesitation and tried to excite it. British Medical Journal 2000; 320: 1512-3. As dog bite is a common problem in Sri Lanka this type of preventive educational strategies are relevant to us. Behaviours that are less likely to provoke an attack are given as asking permission (from the handler), approaching the dog slowly, extending the hand palm down, patting the dog under the chin and on the chest, avoiding eye contact, walking away slowly and quietly. Disturbing even a known friendly dog when it is tied up, sleeping, eating or in a car, are given as behaviours that are more likely to provoke an attack. 


\section{Nebulised corticosteroids in respiratory syncitial virus (RSV) bronchiolitis}

The acute inflammatory changes in the airways of infants with acute bronchiolitis are similar to those seen in acute exacerbations of asthma. Therefore it is possible that corticosteroids administered in the acute phase of bronchiolitis may benefit the natural history. A few studies have reported beneficial effects of steroids given in the acute phase of viral bronchiolitis but the majority of the studies show no clinical benefit. A recent multicentre randomised double blind placebo controlled trial involving 161 infants with viral bronchiolitis has compared the effects of nebulised budesonide on duration of hospital stay, readmission rate, general practice consultation and use of anti-wheeze medication subsequently. It has shown that there are no short term or long term clinical benefits of nebulised corticosteroid administered in the acute phase. Archives of Disease in Childhood 2000; 82: 126-30.

\section{A good death}

In an Editorial in the British Medical Journal, its editor has identified certain principles of a good death. Some of these principles are given below.

- To know when death is coming and to understand what can be expected.

- To be able to retain control of what happens.

- To have good control of symptoms (eg. pain) due to underlying disease.

- To be given dignity and privacy and have choice of where death occurs.

- To have access to information and expertise that may become necessary.

- To have access to spiritual and emotional support required.

- To be able to issue advance directives which ensures that wishes are respected.

- To have time to say goodbye and to have control over other aspects of timing.

- To be able to leave when it is time to go, and not to have life prolonged pointlessly.

British Medical Journal 2000; 320: 129-30. Should we, our relatives and carers plan in advance to achieve many of the above?

R L Jayakody, Senior Lecturer, Department of Pharmacology, Faculty of Medicine, University of Colombo. 